\title{
Extended Mixed Vector Equilibrium Problems
}

\author{
Mijanur Rahaman, ${ }^{1}$ Adem Kılıçman, ${ }^{2}$ and Rais Ahmad ${ }^{1}$ \\ ${ }^{1}$ Department of Mathematics, Aligarh Muslim University, Aligarh 202002, India \\ ${ }^{2}$ Department of Mathematics and Institute for Mathematical Research, Universiti Putra Malaysia, 43400 Serdang, Selangor, Malaysia
}

Correspondence should be addressed to Adem Kılıçman; akilic@upm.edu.my

Received 19 March 2014; Accepted 1 April 2014; Published 27 April 2014

Academic Editor: Jen-Chih Yao

Copyright (C) 2014 Mijanur Rahaman et al. This is an open access article distributed under the Creative Commons Attribution License, which permits unrestricted use, distribution, and reproduction in any medium, provided the original work is properly cited.

We study extended mixed vector equilibrium problems, namely, extended weak mixed vector equilibrium problem and extended strong mixed vector equilibrium problem in Hausdorff topological vector spaces. Using generalized KKM-Fan theorem (Ben-ElMechaiekh et al.; 2005), some existence results for both problems are proved in noncompact domain.

\section{Introduction}

Giannessi [1] first introduced and studied vector variational inequality problem in a finite-dimensional vector space. Since then, the theory with applications for vector variational inequalities, vector equilibrium problems, vector complementarity problems, and many other problems has been extensively studied in a general setting by many authors; see for example [2-7] and references therein.

In 1989, Parida et al. [8] developed a theory for the existence of a solution of variational-like inequality problem and showed the relationship between variational-like inequality problem and a mathematical programming problem. The problem of vector variational-like inequalities is also one of the generalizations of vector variational inequalities studied by many authors; see [9-11] and references therein.

On the other hand, equilibrium problem was first introduced and studied by Blum and Oettli [12]. Many authors [13-15] have proved the existence of equilibrium problems by using different generalization of monotonicity condition and generalized convexity assumption. The main objective of our work is to study an extended weak mixed vector equilibrium problem and an extended strong mixed vector equilibrium problem and we prove existence results for both problems by using a generalized coercivity type condition, namely, coercing family. Both problems are combination of a vector equilibrium problem and a vector variational-like inequality problem. Our results presented in this paper improve and generalize some known results obtained by [12, 16-18].

\section{Preliminaries}

Throughout this paper, let $X$ and $Y$ be the Hausdorff topological vector spaces. Let $K$ be a nonempty convex closed subset of $X$ and $C \subseteq Y$ a pointed closed convex cone with int $C \neq \emptyset$. The partial order " $\leq_{C}$ " on $Y$ induced by $C$ is defined by $x \leq_{C} y$ if and only if $y-x \in C$. Let $f: K \times K \rightarrow Y, T: K \rightarrow L(X, Y)$ and $\eta: K \times K \rightarrow X$ be the mappings, where $L(X, Y)$ is the space of all continuous linear mappings from $X$ to $Y$. We denote the value of $l \in L(X, Y)$ at $x \in K$ by $\langle l, x\rangle$. In this paper, we consider the following problems.

Find $\bar{x} \in K$ such that

$$
\begin{array}{rr}
f(\bar{x}, y)+\langle T(\bar{x}), \eta(y, \bar{x})\rangle \notin-\operatorname{int} C ; & \forall y \in K, \\
f(\bar{x}, y)+\langle T(\bar{x}), \eta(y, \bar{x})\rangle \notin-C \backslash\{0\} ; & \forall y \in K .
\end{array}
$$

We call problem (1) extended weak mixed vector equilibrium problem and problem (2) extended strong mixed vector equilibrium problem.

Let us recall some definitions and results that are needed to prove the main results of this paper. 
Definition 1. A mapping $g: K \rightarrow 2^{Y}$ is said to be

(i) lower semicontinuous with respect to $C$ at a point $x_{0} \in K$, if for any neighborhood $V$ of $g\left(x_{0}\right)$ in $Y$, there exists a neighborhood $U$ of $x_{0}$ in $X$ such that

$$
g(U \cap K) \subseteq V+C
$$

(ii) upper semicontinuous with respect to $C$ at a point $x_{0} \in K$, if

$$
g(U \cap K) \subseteq V-C
$$

(iii) continuous with respect to $C$ at a point $x_{0} \in K$, if it is lower semicontinuous and upper semicontinuous with respect to $C$ at that point.

Remark 2. If $g$ is lower semicontinuous, upper semicontinuous, and continuous with respect to $C$ at any arbitrary point of $K$, then $g$ is lower semicontinuous, upper semicontinuous, and continuous with respect to $C$ on $K$, respectively.

Definition 3 (see [19]). Let $T: K \rightarrow L(X, Y)$ and $\eta: K \times$ $K \rightarrow X$ be the mappings. Then

(i) $T$ is said to be $C$ - $\eta$-pseudomonotone, if for any $x, y \in$ $K$,

$$
\begin{aligned}
& \langle T(x), \eta(y, x)\rangle \notin-\operatorname{int} C \\
& \text { implies }\langle T(y), \eta(y, x)\rangle \notin-\operatorname{int} C ;
\end{aligned}
$$

(ii) $T$ is said to be strongly $C-\eta$-pseudomonotone, if for any $x, y \in K$,

$\langle T(x), \eta(y, x)\rangle \notin-C \backslash\{0\} \quad$ implies $\langle T(y), \eta(y, x)\rangle \in C ;$

(iii) $T$ is $\eta$-hemicontinuous, if for any given $x, y \in K$ and $\lambda \in(0,1]$, the mapping $\lambda \mapsto\langle T(x+\lambda(y-x)), \eta(y, x)\rangle$ is continuous at $0^{+}$;

(iv) $\eta$ is said to be affine in the first argument, if for any $x_{i} \in K$ and $\lambda_{i} \geq 0,1 \leq i \leq n$ with $\sum_{i=1}^{n} \lambda_{i}=1$ and any $y \in K$, we have

$$
\eta\left(\sum_{i=1}^{n} \lambda_{i} x_{i}, y\right)=\sum_{i=1}^{n} \lambda_{i} \eta\left(x_{i}, y\right) .
$$

Definition 4 (see [20]). Consider a subset $K$ of a topological vector space $X$ and a topological space $Y$. A family $\left\{\left(C_{i}, Z_{i}\right)\right\}_{i \in I}$ of pair of sets is said to be coercing for a mapping $F: K \rightarrow 2^{Y}$ if and only if

(i) for each $i \in I, C_{i}$ is contained in a compact convex subset of $K$ and $Z_{i}$ is a compact subset of $Y$;

(ii) for each $i, j \in I$, there exists $k \in I$ such that $C_{i} \cup C_{j} \subseteq$ $C_{k}$;

(iii) for each $i \in I$, there exists $k \in I$ with $\bigcap_{x \in C_{k}} F(x) \subseteq Z_{i}$.
Remark 5. In case where the coercing family reduced to single element, condition (iii) of Definition 4 appeared first in this generality (with two sets $C$ and $Z$ ) in [21] and generalizes the condition of Karamardian [22] and Allen [23]. Condition (iii) is also an extension of coercivity condition given by Fan [24].

Definition 6. Let $K$ be a nonempty convex subset of a topological vector space $X$. A multivalued mapping $G: K \rightarrow$ $2^{X}$ is said to be KKM mapping, if, for every finite subset $\left\{x_{i}\right\}_{i \in I}$ of $K$,

$$
\operatorname{Co}\left\{x_{i}: i \in I\right\} \subseteq \bigcup_{i \in I} F\left(x_{i}\right),
$$

where $\operatorname{Co}\left\{x_{i}: i \in I\right\}$ denotes the convex hull of $\left\{x_{i}\right\}_{i \in I}$ and $I$ is a finite index set.

Theorem 7 (see [20]). Let $X$ be a Hausdorff topological vector space, $Y$ a convex subset of $X, K$ a nonempty subset of $Y$, and $F: K \rightarrow 2^{Y}$ a KKM mapping with compactly closed values in $Y$ (i.e., for all $x \in K, F(x) \cap Z$ is closed for every compact set $Z$ of $Y$ ). If $F$ admits a coercing family, then

$$
\bigcap_{x \in K} F(x) \neq \emptyset
$$

Lemma 8 (see [25]). Let $X$ be a Hausdorff topological space and $\left\{A_{i}\right\}_{i \in I}$ nonempty compact convex subsets of $X$. Then $\operatorname{Co}\left\{A_{i}: i \in I\right\}$ is compact.

\section{Existence Results}

In this section, we first present an existence result for extended weak mixed vector equilibrium problem (1).

Theorem 9. Let $K$ be a nonempty closed convex subset of a Hausdorff topological vector space X, Y a Hausdorff topological vector space, and $C$ a closed convex pointed cone with int $C \neq \emptyset$. Let $f: K \times K \rightarrow Y, T: K \rightarrow L(X, Y)$ and $\eta: K \times K \rightarrow X$ be the mappings satisfying the following conditions:

(i) $f$ is affine in the second argument and continuous in the first argument;

(ii) $f(x, x)=0$, for all $x \in K$;

(iii) $\eta(x, x)=0$ and $\eta(x, y)+\eta(y, x)=0$, for all $x, y \in K$;

(iv) $\eta$ is affine in both arguments and continuous in the second argument;

(v) $T$ is $\eta$-hemicontinuous, C- $\eta$-pseudomonotone, and continuous;

(vi) the mapping $W: K \rightarrow 2^{Y}$, defined by $W=Y \backslash$ $\{-\operatorname{int} C\}$, is upper semicontinuous on $K$;

(vii) there exists a family $\left\{\left(C_{i}, Z_{i}\right)\right\}_{i \in I}$ satisfying conditions (i) and (ii) of Definition 4 and the following condition: for each $i \in I$, there exists $k \in I$ such that

$\left\{x \in K: f(x, y)+\langle T(x), \eta(y, x)\rangle \notin-\operatorname{int} C, \forall y \in C_{k}\right\}$

$\subseteq Z_{i}$ 
Then, there exists a point $\bar{x} \in K$ such that

$$
f(\bar{x}, y)+\langle T(\bar{x}), \eta(y, \bar{x})\rangle \notin-\operatorname{int} C ; \quad \forall y \in K .
$$

For the proof of Theorem 9, we need the following proposition, for which the assumptions remain the same as in Theorem 9.

Proposition 10. The following two problems are equivalent:

(I) find $\bar{x} \in K$ such that $f(\bar{x}, y)+\langle T(\bar{x}), \eta(y, \bar{x})\rangle \notin$ - int $C$, for all $y \in K$;

(II) find $\bar{x} \in K$ such that $f(\bar{x}, y)-\langle T(y), \eta(\bar{x}, y)\rangle \notin$ $-\operatorname{int} C$, for all $y \in K$.

Proof. Suppose that (I) holds. Then for every $y \in K$, we have

$$
f(\bar{x}, y)+\langle T(\bar{x}), \eta(y, \bar{x})\rangle \notin-\operatorname{int} C .
$$

Since $T$ is $C-\eta$-pseudomonotone, from (12) we have

$$
f(\bar{x}, y)+\langle T(y), \eta(y, \bar{x})\rangle \notin-\operatorname{int} C .
$$

Also from assumptions (iii) and (13), we get

$$
f(\bar{x}, y)-\langle T(y), \eta(\bar{x}, y)\rangle \notin-\operatorname{int} C ;
$$

that is, (II) holds.

Conversely, assume that (II) holds for all $y \in K$. Then there exists $\bar{x} \in K$ such that

$$
f(\bar{x}, y)-\langle T(y), \eta(\bar{x}, y)\rangle \notin-\operatorname{int} C .
$$

For a fixed $y \in K$, set $x_{\lambda}=\lambda y+(1-\lambda) \bar{x}$, for $\lambda \in[0,1]$. Obviously, $x_{\lambda} \in K$ and it follows that

$$
f\left(\bar{x}, x_{\lambda}\right)-\left\langle T\left(x_{\lambda}\right), \eta\left(\bar{x}, x_{\lambda}\right)\right\rangle \notin-\operatorname{int} C .
$$

Multiplying $(16)$ by $(1-\lambda)$, we have

$$
(1-\lambda) f\left(\bar{x}, x_{\lambda}\right)-(1-\lambda)\left\langle T\left(x_{\lambda}\right), \eta\left(\bar{x}, x_{\lambda}\right)\right\rangle \notin-\operatorname{int} C .
$$

Since $\eta$ is affine and $\eta(x, x)=0$, we have

$$
\begin{aligned}
0 & =\left\langle T\left(x_{\lambda}\right), \eta\left(x_{\lambda}, x_{\lambda}\right)\right\rangle \\
& =\lambda\left\langle T\left(x_{\lambda}\right), \eta\left(y, x_{\lambda}\right)\right\rangle+(1-\lambda)\left\langle T\left(x_{\lambda}\right), \eta\left(\bar{x}, x_{\lambda}\right)\right\rangle .
\end{aligned}
$$

That is,

$$
-(1-\lambda)\left\langle T\left(x_{\lambda}\right), \eta\left(\bar{x}, x_{\lambda}\right)\right\rangle=\lambda\left\langle T\left(x_{\lambda}\right), \eta\left(y, x_{\lambda}\right)\right\rangle .
$$

Since $(1-\lambda) f\left(\bar{x}, x_{\lambda}\right) \in Y$, adding $(1-\lambda) f\left(\bar{x}, x_{\lambda}\right)$ on both sides of (19), we obtain

$$
\begin{array}{r}
(1-\lambda) f\left(\bar{x}, x_{\lambda}\right)-(1-\lambda)\left\langle T\left(x_{\lambda}\right), \eta\left(\bar{x}, x_{\lambda}\right)\right\rangle \\
\quad=(1-\lambda) f\left(\bar{x}, x_{\lambda}\right)+\lambda\left\langle T\left(x_{\lambda}\right), \eta\left(y, x_{\lambda}\right)\right\rangle .
\end{array}
$$

Combining (17) and (20), we get

$$
(1-\lambda) f\left(\bar{x}, x_{\lambda}\right)+\lambda\left\langle T\left(x_{\lambda}\right), \eta\left(y, x_{\lambda}\right)\right\rangle \notin-\operatorname{int} C .
$$

Since $f$ is affine in the second argument and $f(x, x)=0,(21)$ implies that

$$
\lambda(1-\lambda) f(\bar{x}, y)+\lambda\left\langle T\left(x_{\lambda}\right), \eta\left(y, x_{\lambda}\right)\right\rangle \notin-\operatorname{int} C .
$$

Since $\eta$ is affine and $\eta(x, x)=0$, then from (22) we deduce that

$$
\lambda(1-\lambda) f(\bar{x}, y)+\lambda(1-\lambda)\left\langle T\left(x_{\lambda}\right), \eta(y, \bar{x})\right\rangle \notin-\operatorname{int} C .
$$

Dividing (23) by $\lambda(1-\lambda)$, we have

$$
f(\bar{x}, y)+\left\langle T\left(x_{\lambda}\right), \eta(y, x)\right\rangle \notin-\operatorname{int} C .
$$

Using $\eta$-hemicontinuity of $T$, we get

$$
f(\bar{x}, y)+\langle T(x), \eta(y, x)\rangle \notin-\operatorname{int} C ;
$$

and hence (II) holds.

Proof of Theorem 9. For each $y \in K$, consider the sets

$$
\begin{aligned}
& F_{1}(y)=\{x \in K: f(x, y)-\langle T(y), \eta(x, y)\rangle \notin-\operatorname{int} C\} \\
& F_{2}(y)=\{x \in K: f(x, y)+\langle T(x), \eta(y, x)\rangle \notin-\operatorname{int} C\} .
\end{aligned}
$$

Then $F_{1}(y)$ and $F_{2}(y)$ are nonempty sets, since $y \in F_{1}(y)$ and $y \in F_{2}(y)$.

First, we prove that $F_{1}$ is a KKM mapping. Indeed, assume that $F_{1}$ is not a KKM mapping. Then, there exists finite subset $\left\{y_{i}: i \in I\right\}$ of $K, \lambda_{i} \geq 0$ for each $i \in I$ with $\sum_{i \in I} \lambda_{i}=1$ and $w=\sum_{i \in I} \lambda_{i} y_{i}$ such that

$$
w \notin \bigcup_{i \in I} F_{1}\left(y_{i}\right)
$$

That is,

$$
f\left(w, y_{i}\right)-\left\langle T\left(y_{i}\right), \eta\left(w, y_{i}\right)\right\rangle \in-\operatorname{int} C, \quad \forall i \in I .
$$

As int $C$ is convex, therefore

$$
\sum_{i \in I} \lambda_{i} f\left(w, y_{i}\right)-\sum_{i \in I} \lambda_{i}\left\langle T\left(y_{i}\right), \eta\left(w, y_{i}\right)\right\rangle \in-\operatorname{int} C .
$$

Since $f$ is affine in the second argument and $\eta$ is affine, from (29) we have

$$
\begin{aligned}
f & (w, w)-\left\langle T\left(y_{i}\right), \eta(w, w)\right\rangle \\
& =f\left(w, \sum_{i \in I} \lambda_{i} y_{i}\right)-\left\langle T\left(y_{i}\right), \eta\left(w, \sum_{i \in I} \lambda_{i} y_{i}\right)\right\rangle \\
& =\sum_{i \in I} \lambda_{i} f\left(w, y_{i}\right)-\sum_{i \in I} \lambda_{i}\left\langle T\left(y_{i}\right), \eta\left(w, y_{i}\right)\right\rangle \\
& \in-\operatorname{int} C .
\end{aligned}
$$

By assumptions (ii) and (iii), we know $\eta(x, x)=f(x, x)=$ 0 . Then (30) implies that $0 \in-\operatorname{int} C$, which contradicts the pointedness of $C$ and hence $F_{1}$ is a KKM mapping. 
Further, we prove that

$$
\bigcap_{y \in K} F_{1}(y)=\bigcap_{y \in K} F_{2}(y) .
$$

Let $x \in F_{1}(y)$, so that

$$
f(x, y)-\langle T(y), \eta(x, y)\rangle \notin-\operatorname{int} C .
$$

Since $T$ is $C$ - $\eta$-pseudomonotone and $\eta(x, y)+\eta(y, x)=0$, then (32) implies that

$$
f(x, y)+\langle T(x), \eta(y, x)\rangle \notin-\operatorname{int} C,
$$

and so $x \in F_{2}(y)$ for each $y \in K$; that is, $F_{1}(y) \subseteq F_{2}(y)$ and hence

$$
\bigcap_{y \in K} F_{1}(y) \subseteq \bigcap_{y \in K} F_{2}(y)
$$

Conversely, suppose that $x \in \bigcap_{y \in K} F_{2}(y)$. Then

$$
f(x, y)+\langle T(x), \eta(y, x)\rangle \notin-\operatorname{int} C .
$$

It follows from Proposition 10 that

$$
f(x, y)-\langle T(y), \eta(x, y)\rangle \notin-\operatorname{int} C ;
$$

that is, $x \in F_{1}(y)$ and so

$$
\bigcap_{y \in K} F_{2}(y) \subseteq \bigcap_{y \in K} F_{1}(y) .
$$

Combining (34) and (37), we obtain

$$
\bigcap_{y \in K} F_{1}(y)=\bigcap_{y \in K} F_{2}(y) .
$$

Now, since $F_{1}$ is a KKM mapping, for any finite subset $\left\{y_{i}\right.$ : $i \in I\}$ of $K$, we have

$$
\operatorname{Co}\left\{y_{i}: i \in I\right\} \subseteq \bigcup_{i \in I} F_{1}\left(y_{i}\right) \subseteq \bigcup_{i \in I} F_{2}\left(y_{i}\right)
$$

This implies that $F_{2}$ is also a KKM mapping.

In order to show that $F_{2}(y)$ is closed for all $y \in K$, let us assume that $\left\{x_{\alpha}\right\}$ is a net in $F_{2}(y)$ such that $x_{\alpha} \rightarrow x$. Then

$$
f\left(x_{\alpha}, y\right)+\left\langle T\left(x_{\alpha}\right), \eta\left(y, x_{\alpha}\right)\right\rangle \notin-\operatorname{int} C .
$$

Since $f$ is continuous in the first argument, $\eta$ is continuous in the second argument, and $T$ is continuous, we have

$$
\begin{aligned}
& f\left(x_{\alpha}, y\right)+\left\langle T\left(x_{\alpha}\right), \eta\left(y, x_{\alpha}\right)\right\rangle \\
& \quad \longrightarrow f(x, y)+\langle T(x), \eta(y, x)\rangle .
\end{aligned}
$$

As $W=Y \backslash\{-\operatorname{int} C\}$ is upper semicontinuous, we obtain

$$
f(x, y)+\langle T(x), \eta(y, x)\rangle \in W,
$$

and thus, we have

$$
f(x, y)+\langle T(x), \eta(y, x)\rangle \notin-\operatorname{int} C .
$$

Therefore $x \in F_{2}(y)$, for all $y \in K$ and hence $F_{2}$ is closed. In view of assumption (vii), $F_{2}$ has compactly closed values in K.

By assumption (vii), we see that the family $\left\{\left(C_{i}, Z_{i}\right)\right\}_{i \in I}$ satisfies the condition which is for all $i \in I$ there exists $k \in I$ such that

$$
\bigcap_{y \in C_{k}} F_{2}(y) \subseteq Z_{i}
$$

and consequently, it is a coercing family for $F_{2}$.

Finally, we conclude that $F_{2}$ satisfies all the hypotheses of Theorem 7 and thus we have

$$
\bigcap_{y \in K} F_{2}(y) \neq \emptyset .
$$

Hence, there exists $\bar{x} \in \bigcap_{y \in K} F_{2}(y)$ such that for all $y \in K$

$$
f(\bar{x}, y)+\langle T(\bar{x}), \eta(y, \bar{x})\rangle \notin-\operatorname{int} C .
$$

This completes the proof.

Now, we prove an existence result for extended strong mixed vector equilibrium problem (2).

Theorem 11. Let $f$ and $\eta$ satisfy the assumptions (i)-(iv) of Theorem 9. In addition, assume that the following conditions are satisfied:

(v)' for each $y \in K$, the set $\{x \in K: f(x, y)+$ $\langle T(x), \eta(y, x)\rangle \in-C \backslash\{0\}\}$ is open in $K$;

(vi)' there exists a nonempty compact and convex subset $D$ of $K$ and, for each $x \in K \backslash D$, there exists $u \in D$ such that

$$
f(x, u)+\langle T(x), \eta(u, x)\rangle \in-C \backslash\{0\} ;
$$

(vii) $^{\prime}$ there exists a family $\left\{\left(C_{i}, Z_{i}\right)\right\}_{i \in I}$ satisfying conditions (i) and (ii) of Definition 4 and the following condition which is for each $i \in I$ there exists $k \in I$ such that

$$
\begin{aligned}
\{x & \left.\in K: f(x, y)+\langle T(x), \eta(y, x)\rangle \notin-C \backslash\{0\}, \forall y \in C_{k}\right\} \\
& \subseteq Z_{i} .
\end{aligned}
$$

Then, there exists a point $\bar{x} \in K$ such that for all $y \in K$

$$
f(\bar{x}, y)+\langle T(\bar{x}), \eta(y, \bar{x})\rangle \notin-C \backslash\{0\} .
$$

Proof. Let $F: K \rightarrow 2^{D}$ be defined by

$$
F(y)=\{x \in D: f(x, y)+\langle T(x), \eta(y, x)\rangle \notin-C \backslash\{0\}\},
$$$$
\forall y \in K \text {. }
$$ 
Obviously, for all $y \in K$

$$
F(y)=\{x \in K: f(x, y)+\langle T(x), \eta(y, x)\rangle \notin-C \backslash\{0\}\}
$$

$\cap D$.

As $F(y)$ is closed subset of $D$ and $D$ is compact, therefore $F(y)$ is compactly closed.

Now, we show that, for any finite set $\left\{y_{i}\right\}_{i \in I}$ of $K$, $\bigcap_{i \in I} F\left(y_{i}\right) \neq \emptyset$. For this, let $E=\operatorname{Co}\left\{D \cup\left\{y_{i}\right\}_{i \in I}\right\}$. Then, by Lemma $8, E$ is a compact and convex subset of $K$.

Let $G: E \rightarrow 2^{E}$ be defined by

$$
G(y)=\{x \in E: f(x, y)+\langle T(x), \eta(y, x)\rangle \notin-C \backslash\{0\}\},
$$

First, we prove that $G$ is a KKM mapping. On contrary, suppose that $G$ is not a KKM mapping; then there exists $v \in \operatorname{Co}\left\{y_{i}\right\}_{i \in I}$ such that, for $\lambda_{i} \geq 0$ with $\sum_{i \in I} \lambda_{i}=1$, we have

$$
v=\sum_{i \in I} \lambda_{i} y_{i} \notin \bigcup_{i \in I} G\left(y_{i}\right) \text {, }
$$

which implies

$$
f\left(v, y_{i}\right)+\left\langle T(v), \eta\left(y_{i}, v\right)\right\rangle \in-C \backslash\{0\} .
$$

Since $f$ and $\eta$ are affine in the second argument, (54) implies that

$$
\begin{aligned}
f(v, v) & +\langle T(v), \eta(v, v)\rangle \\
= & f\left(v, \sum_{i \in I} \lambda_{i} y_{i}\right)+\left\langle T(v), \eta\left(\sum_{i \in I} \lambda_{i} y_{i}, v\right)\right\rangle \\
= & \sum_{i \in I} \lambda_{i} f\left(v, y_{i}\right)+\sum_{i \in I} \lambda_{i}\left\langle T(v), \eta\left(y_{i}, v\right)\right\rangle \\
= & \sum_{i \in I} \lambda_{i}\left\{f\left(v, y_{i}\right)+\left\langle T(v), \eta\left(y_{i}, v\right)\right\rangle\right\} \\
& \in-C \backslash\{0\} .
\end{aligned}
$$

Since $f(x, x)=\eta(x, x)=0,(55)$ implies that $0 \in-C \backslash\{0\}$, which is a contradiction. Hence, $G$ is a KKM mapping.

As $G(y)$ is closed subset of $E$, therefore it is compactly closed. From assumption (vii) ${ }^{\prime}$, it is clear that the family $\left\{C_{i}, Z_{i}\right\}_{i \in I}$ satisfies the condition $\bigcap_{y \in C_{k}} G(y) \subseteq Z_{i}$ and therefore it is a coercing family for $G$. Applying Theorem 7, we obtain

$$
\bigcap_{y \in E} G(y) \neq \emptyset .
$$

Thus we conclude that there exists $y_{0} \in \bigcap_{y \in E} G(y)$.

To show that $y_{0} \in D$, on contrary suppose that $y_{0} \in E \backslash D$. Then condition (vi)' implies that there exists $u \in D$ such that

$$
f\left(y_{0}, u\right)+\left\langle T\left(y_{0}\right), \eta\left(u, y_{0}\right)\right\rangle \in C \backslash\{0\},
$$

which contradicts the fact that $y_{0} \in G(y)$, and hence $y_{0} \in D$. Since $F\left(y_{i}\right)=G\left(y_{i}\right) \cap D$, for each $y_{i} \in E$, it follows that $y_{0} \in$ $\bigcap_{i \in I} F\left(y_{i}\right)$; that is, $\bigcap_{i \in I} F\left(y_{i}\right) \neq \emptyset$, for finite subset $\left\{y_{i}\right\}_{i \in I} \subset K$. As $F(y)$ is closed and compact, it follows that, for each $y \in K$, there exists $\bar{x} \in D$ such that $\bar{x} \in \bigcap_{y \in K} F(y)$. Hence, there exists $\bar{x} \in K$ such that, for all $y \in K$,

$$
f(\bar{x}, y)+\langle T(\bar{x}), \eta(y, \bar{x})\rangle \notin-C \backslash\{0\} .
$$

This completes the proof.

Theorem 12. Let the assumptions (i)-(iv) of Theorem 9 hold. In addition, we assume that $T$ is strongly $C-\eta$-pseudomonotone and $\eta$-hemicontinuous. Then the following problems are equivalent:

(I) find $\bar{x} \in K$ such that $f(\bar{x}, y)+\langle T(\bar{x}), \eta(y, \bar{x})\rangle \notin-C \backslash$ $\{0\}$, for all $y \in K$;

(II) find $\bar{x} \in K$ such that $f(\bar{x}, y)+\langle T(y), \eta(y, \bar{x})\rangle \in$ $C$, for all $y \in K$.

Proof. Suppose (I) holds. By using the definition of strong $C$ $\eta$-pseudomonotonicity of $T$, (II) follows directly.

Conversely, suppose (II) holds for all $y \in K$. Then we can find $\bar{x} \in K$ such that

$$
f(\bar{x}, y)+\langle T(y), \eta(y, \bar{x})\rangle \in C .
$$

By substituting $x_{\lambda}=\bar{x}+\lambda(y-\bar{x})$, for $\lambda \in[0,1]$, in (59), we obtain

$$
f\left(\bar{x}, x_{\lambda}\right)+\left\langle T\left(x_{\lambda}\right), \eta\left(x_{\lambda}, \bar{x}\right)\right\rangle \in C .
$$

As $\eta$ is affine and $\eta(x, x)=0,(60)$ implies that

$$
f\left(\bar{x}, x_{\lambda}\right)+\lambda\left\langle T\left(x_{\lambda}\right), \eta(y, \bar{x})\right\rangle \in C .
$$

Since $f$ is affine in the second argument and $f(x, x)=0$, from (61) we get

$$
\lambda f(\bar{x}, y)+\lambda\left\langle T\left(x_{\lambda}\right), \eta(y, \bar{x})\right\rangle \in C .
$$

As $C$ is a cone, therefore

$$
f(\bar{x}, y)+\left\langle T\left(x_{\lambda}\right), \eta(y, \bar{x})\right\rangle \in C .
$$

On contrary suppose that

$$
\left\{f(\bar{x}, y)+\left\langle T\left(x_{\lambda}\right), \eta(y, \bar{x})\right\rangle\right\} \cap(Y \backslash C) \neq \emptyset .
$$

As $T$ is $\eta$-hemicontinuous, we have

$$
\{f(\bar{x}, y)+\langle T(\bar{x}), \eta(y, \bar{x})\rangle\} \cap(Y \backslash C) \neq \emptyset,
$$

for sufficiently small $\lambda$, which contradicts (63). Therefore we have

$$
f(\bar{x}, y)+\langle T(\bar{x}), \eta(y, \bar{x})\rangle \notin-C \backslash\{0\},
$$

and hence (I) holds. This completes the proof. 


\section{Conflict of Interests}

The authors declare that there is no conflict of interests regarding the publication of this paper.

\section{Acknowledgment}

The authors gratefully acknowledge that this research was partially supported by the Universiti Putra Malaysia under GP-IBT Grant Scheme having Project no. GPIBT/2013/9420100.

\section{References}

[1] F. Giannessi, "Theorems of alternative, quadratic programs and complementarity problems," in Variational Inequalities and Complementarity Problems, R.W. Cottle, F. Giannessi, and J.L. Lions, Eds., pp. 151-186, John Wiley \& Sons, New York, NY, USA, 1980.

[2] G.-Y. Chen, X. X. Huang, and X. Q. Yang, Vector Optimization: Set-Valued and Variational Analysis, vol. 541 of Lecture Notes in Economics and Mathematical Systems, Springer, Berlin, Germany, 2005.

[3] Y.-P. Fang and N.-J. Huang, "Feasibility and solvability of vector variational inequalities with moving cones in Banach spaces," Nonlinear Analysis. Theory, Methods \& Applications A: Theory and Methods, vol. 70, no. 5, pp. 2024-2034, 2009.

[4] F. Giannessi, Vector Variational Inequalities and Vector Equilibrium, Nonconvex Optimization and Its Applications, vol. 38, Kluwer Academic, Dordrecht, The Netherlands, 2000.

[5] N. J. Huang, A. M. Rubinov, and X. Q. Yang, "Vector optimization problems with nonconvex preferences," Journal of Global Optimization, vol. 40, no. 4, pp. 765-777, 2008.

[6] G. Isac, V. A. Bulavsky, and V. V. Kalashnikov, Complementarity, Equilibrium, Efficiency and Economics, Nonconvex Optimization and Its Applications, vol. 63, Kluwer Academic Publishers, Dordrecht, The Netherlands, 2002.

[7] J. Li, N.-j. Huang, and J. K. Kim, "On implicit vector equilibrium problems," Journal of Mathematical Analysis and Applications, vol. 283, no. 2, pp. 501-512, 2003.

[8] J. Parida, M. Sahoo, and A. Kumar, "A variational-like inequality problem," Bulletin of the Australian Mathematical Society, vol. 39, no. 2, pp. 225-231, 1989.

[9] M. F. Khan and Salahuddin, "On generalized vector variationallike inequalities," Nonlinear Analysis. Theory, Methods \& Applications A: Theory and Methods, vol. 59, no. 6, pp. 879-889, 2004.

[10] B. S. Lee, G. M. Lee, and D. S. Kim, "Generalized vector variational-like inequalities on locally convex Hausdorff topological vector spaces," Indian Journal of Pure and Applied Mathematics, vol. 28, no. 1, pp. 33-41, 1997.

[11] A. H. Siddiqi, M. F. Khan, and Salahuddin, "On vector variational-like inequalities," Far East Journal of Mathematical Sciences, vol. 3, pp. 319-329, 1998.

[12] E. Blum and W. Oettli, "From optimization and variational inequalities to equilibrium problems," The Mathematics Student, vol. 63, no. 1-4, pp. 123-145, 1994.

[13] M. Bianchi, N. Hadjisavvas, and S. Schaible, "Vector equilibrium problems with generalized monotone bifunctions," Journal of Optimization Theory and Applications, vol. 92, no. 3, pp. 527-542, 1997.
[14] M. Bianchi and S. Schaible, "Equilibrium problems under generalized convexity and generalized monotonicity," Journal of Global Optimization, vol. 30, no. 2-3, pp. 121-134, 2004.

[15] Y. P. Fang and N. J. Huang, "Variational-like inequalities with generalized monotone mappings in Banach spaces," Journal of Optimization Theory and Applications, vol. 118, no. 2, pp. 327338, 2003.

[16] K. Fan, "A minimax inequality and applications," in Inequalities, O. Shisha, Ed., vol. 3, pp. 103-113, Academic Press, New York, NY, USA, 1972.

[17] B.-S. Lee, M. F. Khan, and Salahuddin, "Generalized vector variational-type inequalities," Computers \& Mathematics with Applications, vol. 55, no. 6, pp. 1164-1169, 2008.

[18] Z. Al-Rumaih, S. Chebbi, and H. K. Xu, "Noncompact equilibrium points and applications," Journal of Applied Mathematics, vol. 2012, Article ID 373462, 9 pages, 2012.

[19] S. Kum and M.-M. Wong, "An extension of a generalized equilibrium problem," Taiwanese Journal of Mathematics, vol. 15, no. 4, pp. 1667-1675, 2011.

[20] H. Ben-El-Mechaiekh, S. Chebbi, and M. Florenzano, "A generalized KKMF principle," Journal of Mathematical Analysis and Applications, vol. 309, no. 2, pp. 583-590, 2005.

[21] H. Ben-El-Mechaiekh, P. Deguire, and A. Granas, "Une alternative non linéaire en analyse convexe et applications," Comptes Rendus des Séances de l'Académie des Sciences. Série I. Mathématique, vol. 295, no. 3, pp. 257-259, 1982.

[22] S. Karamardian, “Generalized complementarity problem," Journal of Optimization Theory and Applications, vol. 8, pp. 161-168, 1971.

[23] G. Allen, "Variational inequalities, complementarity problems, and duality theorems," Journal of Mathematical Analysis and Applications, vol. 58, no. 1, pp. 1-10, 1977.

[24] K. Fan, "Some properties of convex sets related to fixed point theorems," Mathematische Annalen, vol. 266, no. 4, pp. 519-537, 1984.

[25] A. E. Taylor, An Introduction to Functional Analysis, John Wiley and Sons, New York, NY, USA, 1963. 


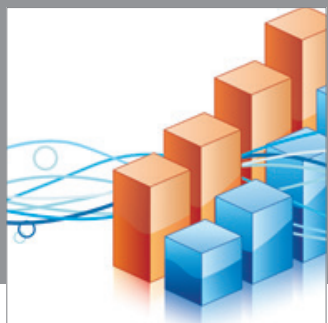

Advances in

Operations Research

mansans

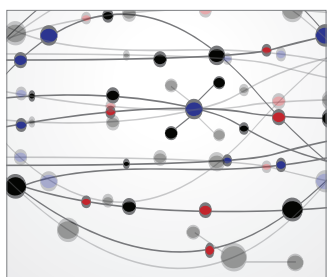

The Scientific World Journal
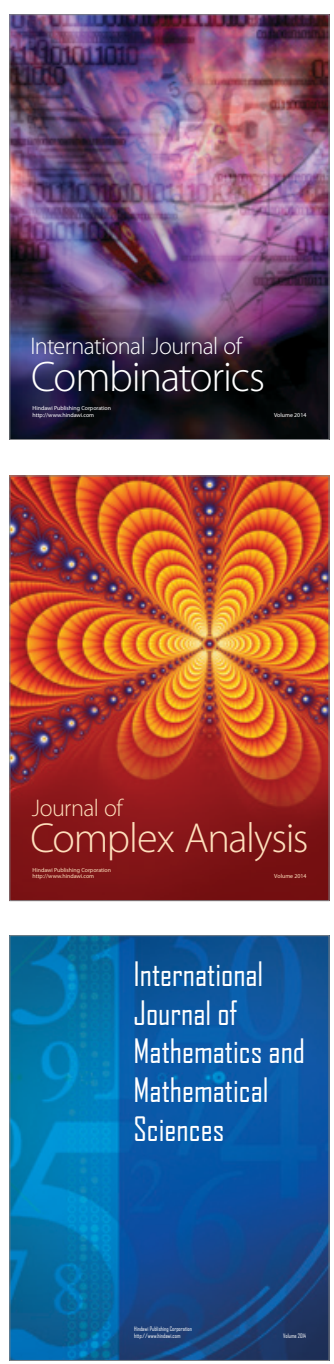
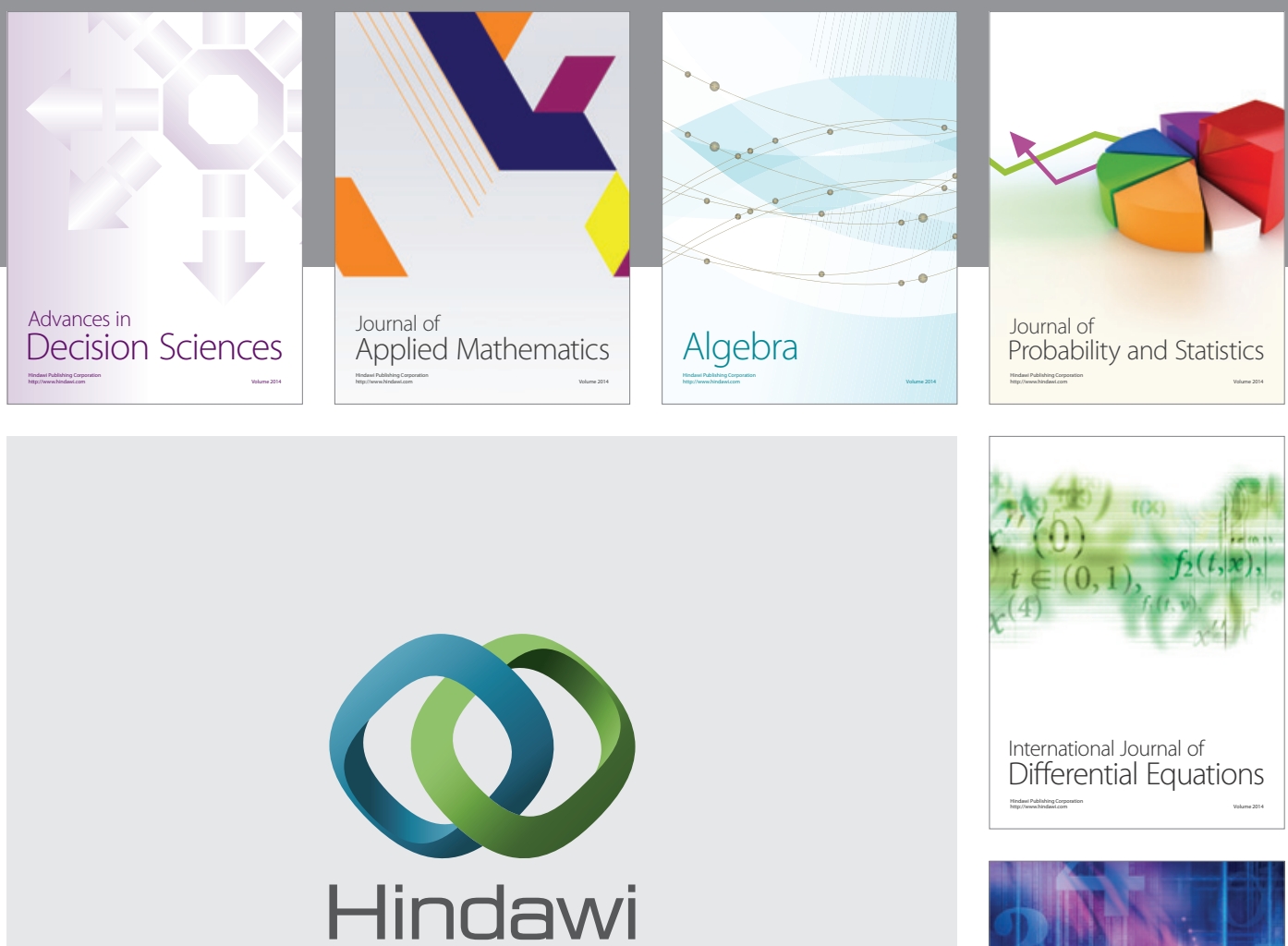

Submit your manuscripts at http://www.hindawi.com
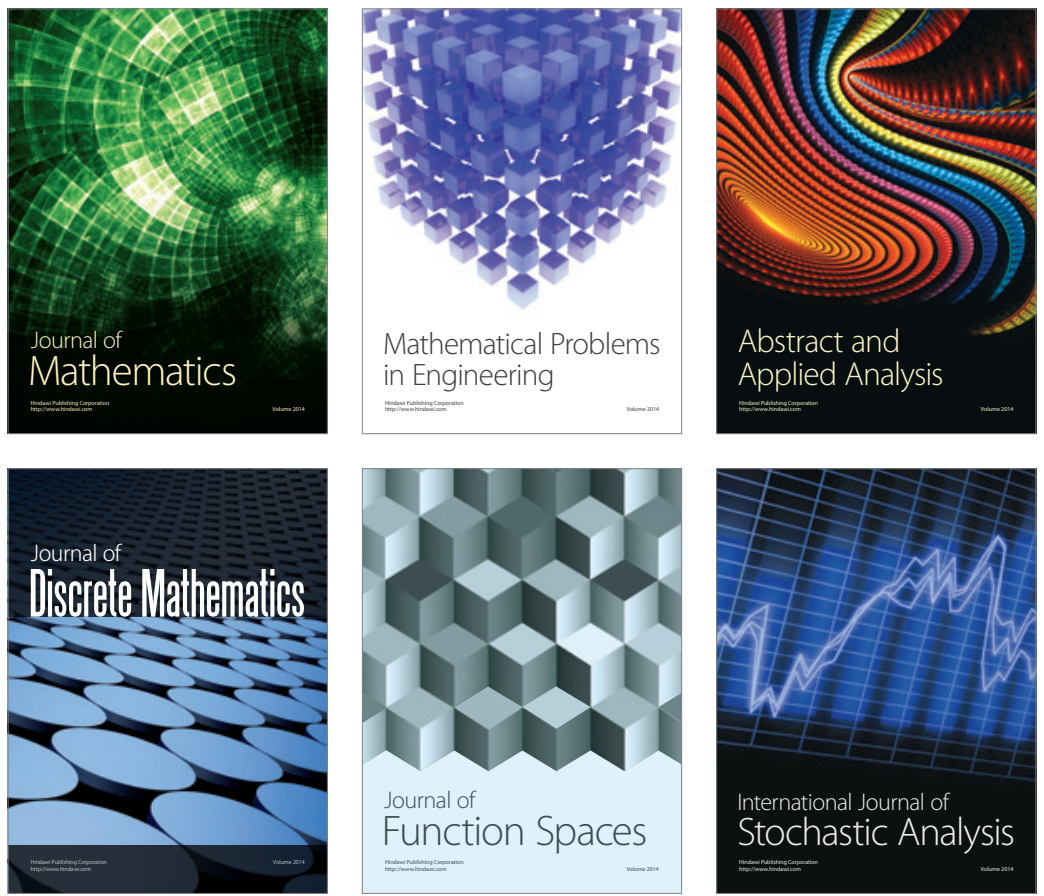

Journal of

Function Spaces

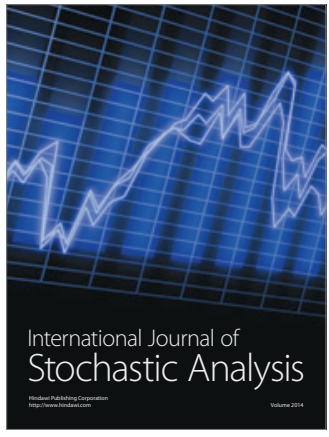

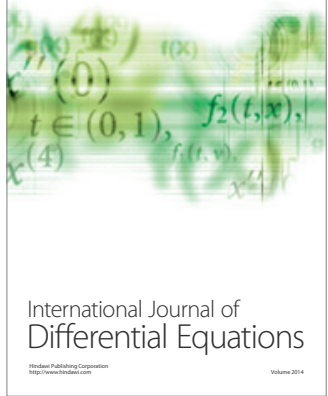
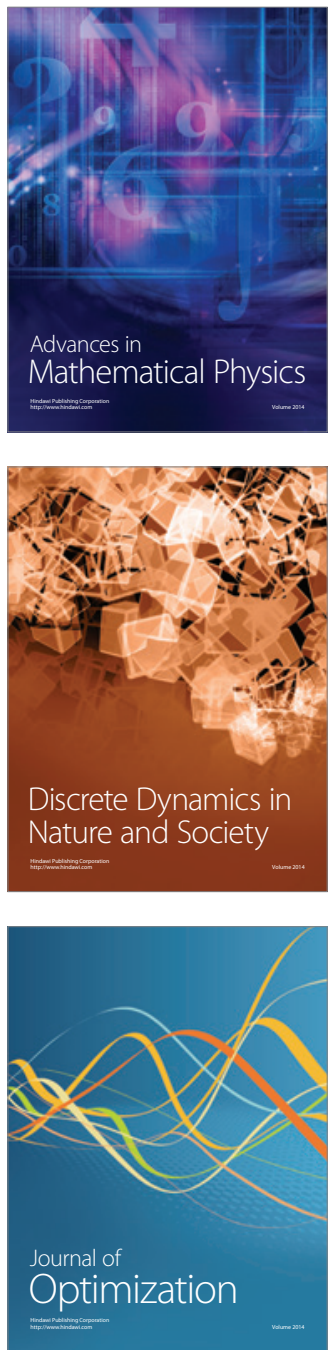